\title{
Sincronismo em um Modelo com Multi-Espécies
}

\author{
Vanderlei Manica, Jacques A. L. Silva, \\ Departamento de Matemática Pura e Aplicada, PPGMAp, UFRGS, \\ 91509-900, Porto Alegre, RS \\ E-mail: vanderlei.manica@ufrgs.br, jaqx@mat.ufrgs.br.
}

\begin{abstract}
Resumo: Apresentamos um modelo metapopulacional composto por sítios distribuídos em duas escalas geográficas e habitados por multi-espécies. Na escala local, os sítios estão acoplados por processo de curta dispersão formando um grupo de sítios, ou conglomerados. Na escala regional, consideramos grupos de sitios conectados por processo de longa dispersão. Analisamos a sincronização do modelo e apresentamos um critério analítico para a sincronização onde todos os grupos de sítios evoluem com a mesma densidade. Através de simulações numéricas, discutimos os diferentes modos de sincronização que dependem de como os indivíduos se distribuem nos sítios que compõem um conglomerado.
\end{abstract}

Palavras-chave: Metapopulação, sincronização, multi-espécies, curta e longa dispersão.

\section{Introdução}

A maior parte da teoria e aplicações em metapopulação considera uma única espécie $[2,8]$. Entretanto, modelos reais com sítios conterão uma coleção de espécies. A dinâmica individual de cada espécie pode apresentar interações biológicas como competição, predação, parasitismo que podem afetar a composição e estabilidade da metapopulação [6, 8, 9]. Em [6] foi analisado a estabilidade local de equilíbrios homogêneos (todas as populações locais tem a mesma densidade a cada geração) e concluíram que a sua estabilidade local depende dos autovalores da matriz que representa o agrupamento dos sítios. Um resultado obtido em [8] é que a dispersão não possui efeito na estabilidade de equilíbrios homogêneos quando a dinâmica local de cada sítio é dada por uma simples espécie ou até mesmo por espécies que competem [8]. Por outro lado, se a dinâmica local é dada por um modelo hospedeiro-parasitóide com taxas de dispersão extremas (uma alta e a outra baixa), a dispersão pode causar instabilidades [9].

Neste trabalho descrevemos um modelo metapopulacional com multi-espécies que habitam sítios distribuídos em duas escalas geográficas. Na seção 2, apresentamos o modelo de equações discretas. Na seção 3, analisamos a estabilidade local de trajetórias sincronizadas e obtemos um critério baseado no cálculo dos números transversais de Lyapunov. Na seção 4, descrevemos as simulações numéricas e comentários finais são feitos na seção 5 .

\section{Modelo Metapopulacional}

Iniciamos descrevendo o modelo visto na escala local, ou seja, a unidade básica é um sítio e indivíduos dispersam entre os sítios que compõem um grupo. Em seguida, consideramos o modelo visto de uma escala regional, ou seja, a unidade básica é um grupo de sítios e os indivíduos migram entre esses grupos.

\subsection{Modelo Metapopulacional Local}

Iniciamos descrevendo o modelo visto da escala local. Assumimos $d$ sítios enumerados por $1,2, \ldots, d$ onde $p$ espécies interagem e conectados por processo de curta dispersão. A dinâmica 
local de cada sítio é dada por um mapeamento $F: R^{p} \rightarrow R^{p}$ de classe $C^{1}$. Na falta de dispersão entre os sítios, a evolução da população é dada por

$$
\mathbf{x}_{t+1}^{i}=F\left(\mathbf{x}_{t}^{i}\right),
$$

onde $\mathbf{x}_{t}^{i}=\left(x_{t}^{i, 1}, x_{t}^{i, 2}, \ldots, x_{t}^{i, p}\right) \in R^{p}$ é o vetor de densidade populacional do sítio $i$ e cada $x_{t}^{i, q}$ representa a densidade de indivíduos da espécie $q, q=1,2, \ldots, p$, no sítio $i, i=1,2, \ldots, d$, no passo de tempo $t$. A função $F$ resulta no número de indivíduos da espécie $q$ após a interação com as outras espécies e pode ser dada por modelos conhecidos da literatura como interação presapredador, interação hospedeiro-parasitóide, competição de espécies ou sistemas epidemiológicos.

Após a dinâmica local dada por (1), indivíduos migram entre esses sítios. Seja $m_{q}$ a fração de indivíduos da espécie $q$ que parte do habitat $i, 0 \leq m_{q} \leq 1, q=1,2, \ldots, p$. A densidade de indivíduos que parte do síto $i$ no passo de tempo $t$ é dado por $\mathbf{M}_{E L} F\left(\mathbf{x}_{t}^{i}\right)$, onde $\mathbf{M}_{E L}=$ $\operatorname{diag}\left(m_{1}, m_{2}, \ldots, m_{p}\right), i=1,2, \ldots, d$. Assim como no caso de uma simples espécie, o processo de migração entre os sítios é descrito por uma matriz de configuração $\Gamma$ com entradas $\gamma_{i k}$ que representa a preferência de indivíduos que partem do sítio $k$ se estabelecer no sítio $i, 0 \leq$ $\gamma_{i k} \leq 1, i, k=1,2, \ldots, d$. Fazendo essas considerações, o número de indivíduos que partem do sítio $i$ e movem-se para o sítio $k$ é $\gamma_{i k} \mathbf{M}_{E L} F\left(\mathbf{x}_{t}^{i}\right)$. Supomos que indivíduos não retornam ao sítio original, portanto $\gamma_{i i}=0$ para todo $i=1,2, \ldots, d$. Além disso, com a suposição na conservação de indivíduos durante a dispersão, temos $\sum_{i=1}^{d} \gamma_{i k}=1$, para todo $k=1,2, \ldots, d$. Portanto, podemos escrever as equações descrevendo a dinâmica do sistema de sítios conectados por processo de curta dispersão e com multi-espécies por

$$
\mathbf{x}_{t+1}^{i}=\left(I-\mathbf{M}_{E L}\right) F\left(\mathbf{x}_{t}^{i}\right)+\sum_{k=1}^{d} \gamma_{i k} \mathbf{M}_{E L} F\left(\mathbf{x}_{t}^{k}\right), i=1,2, \ldots, d .
$$

\subsection{Modelo Metapopulacional Regional}

O modelo metapopulacional em duas escalas geográficas considera grupos de sítios com multiespécies e conectados por processo de longa dispersão. Em cada grupo, os sítios estão conectados por processo de curta dispersão. Denotamos por $x_{t}^{j i, q}$ o número de indivíduos no sítio $i$ do grupo $j$ da espécie $q, i=1,2, \ldots, d, j=1,2, \ldots, n, q=1,2, \ldots, p$, no tempo $t$. A distribuição de indivíduos em cada grupo de sítio $j$ é descrita pelo vetor de dimensão $d p$ dado por $X_{t}^{j}=$ $\left(\mathbf{x}_{t}^{j 1}, \mathbf{x}_{t}^{j 2}, \ldots, \mathbf{x}_{t}^{j d}\right) \in R^{d p}$, onde $\mathbf{x}_{t}^{j i}=\left(x_{t}^{j i, 1}, x_{t}^{j i, 2}, \ldots, x_{t}^{j i, 2}\right) \in R^{p}$. Supomos que indivíduos migram entre os sítios de cada grupo antes do processo de longa dispersão. Portanto, movimentos locais precedem dispersão regional. Definimos a seguinte função vetorial $\mathbf{F}: R^{d p} \rightarrow R^{d p}$ para descrever o processo de dinâmica local em cada grupo de sítio $j$

$$
\mathbf{F}\left(X_{t}^{j}\right)=\left(\begin{array}{c}
\left(I-\mathbf{M}_{E L}\right) F\left(\mathbf{x}_{t}^{j 1}\right)+\sum_{k=1}^{d} \gamma_{1 k} \mathbf{M}_{E L} F\left(\mathbf{x}_{t}^{j k}\right) \\
\left(I-\mathbf{M}_{E L}\right) F\left(\mathbf{x}_{t}^{j 2}\right)+\sum_{k=1}^{d} \gamma_{2 k} \mathbf{M}_{E L} F\left(\mathbf{x}_{t}^{j k}\right) \\
\vdots \\
\left(I-\mathbf{M}_{E L}\right) F\left(\mathbf{x}_{t}^{j d}\right)+\sum_{k=1}^{d} \gamma_{d k} \mathbf{M}_{E L} F\left(\mathbf{x}_{t}^{j k}\right)
\end{array}\right) .
$$

Após ocorrer a dinâmica local em cada grupo de sítios, indivíduos migram entre os grupos por processo de longa dispersão. Seja $\mu_{i, q}$ a fração de indivíduos da espécie $q$ que parte do sítio $i$ de qualquer grupo em um movimento de longa dispersão e se estabelece no grupo vizinho. Consideramos que os indivíduos de cada espécie migram para os grupos vizinhos e se distribuem com diferentes probabilidades nos sítios locais. Indivíduos podem migrar para um grupo vizinho e ter uma preferência para se estabelecerem em determinados sítios, ou indivíduos podem escolher os sítios locais no grupo vizinho com a mesma probabilidade. De modo a ter essa consideração no modelo, definimos o coeficiente de interação por $w_{k i}^{q}, 0 \leq w_{k i}^{q} \leq 1$, para todo $i, k=1,2, \ldots, d$ e $q=1,2, \ldots, p$. Esse coeficiente representa o percentual de indivíduos da espécie $q$ que parte do sítio $i$ num determinado grupo e se estabelece no sítio $k$ do grupo vizinho. 
Portanto, indivíduos que chegam em um novo grupo se distribuem nos $d$ sítios em proporções dadas por $w_{1 i}^{q}, w_{2 i}^{q}, \ldots, w_{d i}^{q}$, onde $\sum_{k=1}^{d} w_{k i}^{q}=1$ (ver Figura 1 ).

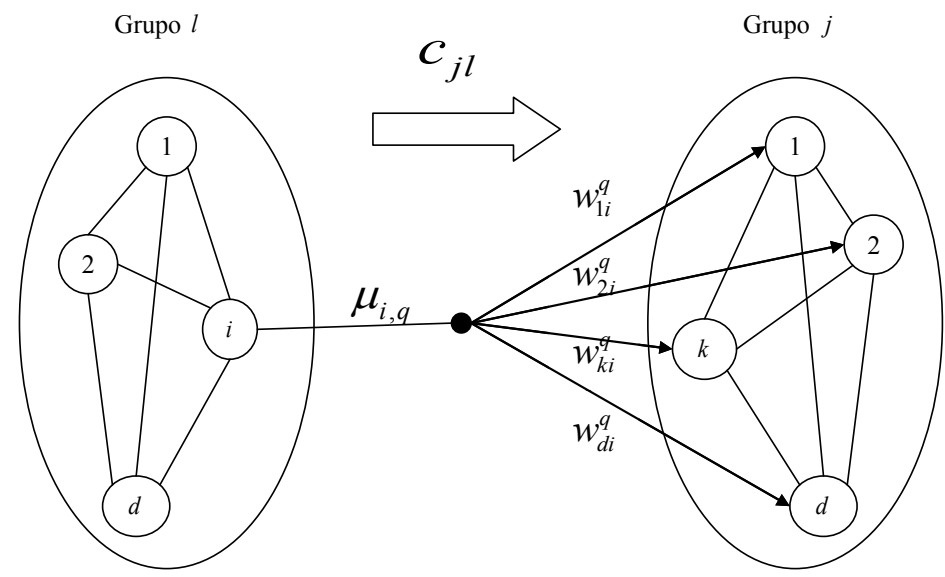

Figura 1: Distribuição de indivíduos de uma determinada espécie entre os grupos. Uma fração de indivíduos $\mu_{i, q}$ da espécie $q$ parte do sítio $i$ de um grupo $l$ e uma fração $c_{j l}$ migra para o grupo vizinho $j$. Os indivíduos que chegam em $l$ se distribuem nos $d$ sítios com proporções $w_{k i}^{q}$. A fração de indivíduos que parte do sítio $i$ do grupo $l$ e chega no sítio $k$ do grupo $j$ é $w_{k i}^{q} c_{j l} \mu_{i, q}$.

Fazendo essas considerações, a fração de indivíduos que parte do sítio $i$ no grupo $l$ e chega no sítio $k$ do grupo $j$ é dada por $w_{k i}^{q} c_{j l} \mu_{i, q}$. Portanto, podemos escrever um sistema de equações descrevendo a dinâmicas do modelo metapopulacional de sítios com multi-espécies e distribuídos em duas escalas como

$$
X_{t+1}^{j}=\left(I-\mathbf{M}_{E G}\right) \mathbf{F}\left(X_{t}^{j}\right)+\sum_{l=1}^{n} c_{j l} W \mathbf{M}_{E G} \mathbf{F}\left(X_{t}^{l}\right), \quad j=1,2, \ldots, n .
$$

onde $\mathbf{M}_{E G}$ é uma matriz de dimensão $d p \times d p$ dada por $\operatorname{diag}\left(\mathbf{M}_{1}, \mathbf{M}_{2}, \ldots, \mathbf{M}_{d}\right)$, onde cada $\mathbf{M}_{i}$ é matriz de dimensão $p \times p$ dada por $\operatorname{diag}\left(\mu_{i, 1}, \mu_{i, 2}, \ldots, \mu_{i, p}\right)$. A matriz $W$ de dimensão $d p \times d p$ possui entradas dadas por $W_{i k}$, tal que $W_{i k}=\operatorname{diag}\left(w_{i k}^{1}, w_{i k}^{2}, \ldots, w_{i k}^{p}\right)$, para todo $i, k=1,2, \ldots, d$. As entradas $W_{i k}$ da matriz $W$ representam a preferência de cada espécie se estabelecer num sítio do grupo vizinho.

A matriz $W$ é denominada matriz de distribuição e diz como os indivíduos se distribuem nos grupos de chegada. A soma de uma coluna de $W$ deve ser zero ou 1 . No caso da soma da coluna de $W$ ser zero significa que a espécie do correspondente sítio não contribui com a dispersão. No caso de ocorrer dispersão, a coluna de $W$ deverá somar 1 . Uma distribuição uniforme é obtida no caso de todas as entradas da diagonal de $W_{i k}$ serem iguais a $\frac{1}{d}$. Observe que essas considerações sobre como migrantes se distribuem no grupo de origem e de chegada valem independentemente de quais são os grupos de origem e chegada. Modelos mais realísticos poderiam incluir tal dependência, portanto, poderíamos considerar uma família de matrizes de distribuição $W_{j l}, j \neq l=1,2, \ldots, n$. Em tais modelos, as unidades básicas não seriam todas iguais e, portanto, faltariam algumas simetrias na rede para garantir a existência de sincronismo, que será discutido a seguir.

\section{Sincronização e Estabilidade Transversal do Modelo Regional}

A seguir, obtemos um critério para a estabilidade assintótica local de soluções sincronizadas baseado no cálculo dos números transversais de Lyapunov. Consideramos que sincronização ocorre se a densidade de todos os grupos é a mesma, ou seja, $X_{t}^{j}=X_{t}^{s}, j=1,2, \ldots, n$, onde $X_{t}^{s}=\left(\mathbf{x}_{t}^{1}, \mathbf{x}_{t}^{2}, \ldots, \mathbf{x}_{t}^{d}\right) \in R^{d p}$, e cada $\mathbf{x}_{t}^{i}=\left(x_{t}^{i, 1}, x_{t}^{i, 2}, \ldots, x_{t}^{i, 2}\right) \in R^{p}, t=0,1,2, \ldots$. Substituindo 
$X_{t}^{j}=X_{t}^{s}, j=1,2, \ldots, n$, na equação (4), obtemos a existência de tais soluções se $\sum_{l=1}^{n} c_{j l}=1$. Além disso, a dinâmica de cada grupo no estado sincronizado satisfaz

$$
X_{t+1}^{s}=\left(I-\mathbf{M}_{E G}+W \mathbf{M}_{E G}\right) \mathbf{F}\left(X_{t}^{s}\right) .
$$

O conjunto solução de (5) representa o estado sincronizado e depende da dinâmica local de cada grupo e do processo de dispersão. Para analisarmos a estabilidade assintótica do estado sincronizado, supomos que a matriz $C$ é diagonizável e decompomos a matriz Jacobiana de (4) em blocos. Linearizando o sistema de equações (4) em torno do estado sincronizado, obtemos

$$
\Delta_{t+1}=J\left(\mathbf{z}_{t}^{s}\right) \Delta_{t}
$$

onde $\Delta_{t} \in R^{n d p}$ é a perturbação do estado sincronizado, $\mathbf{z}_{t}^{s}=\left(X_{t}^{s}, \ldots, X_{t}^{s}\right) \in R^{n d p}$ é a trajetória sincronizada, e $J\left(\mathbf{z}_{t}^{s}\right)$ é a matriz Jacobiana de (4) aplicada em $\mathbf{z}_{t}^{s}$ que pode ser escrita como

$$
J\left(\mathbf{z}_{t}^{s}\right)=\left(I-\mathbf{M}_{E G}\right) \otimes D \mathbf{F}\left(X_{t}^{s}\right)+\left(C \otimes W \mathbf{M}_{E G}\right) D \mathbf{F}\left(X_{t}^{s}\right),
$$

onde $\otimes$ representa o produto de Kronecker.

Supondo que a matriz $C$ é diagonalizável, existe uma matriz inversível $Q$ tal que $\Lambda^{*}=$ $Q C Q^{-1}$, onde $\Lambda^{*}=\operatorname{diag}\left(\lambda_{0}, \lambda_{1}, \ldots, \lambda_{n-1}\right)$, e $\lambda_{j}$ são os autovalores de $C, j=0,1, \ldots, n-1$. Fazendo a mudança de variáveis $Y_{t}=(Q \otimes I) \Delta_{t}$, e considerando (6) e (7), temos

$$
\begin{aligned}
Y_{t+1} & =(Q \otimes I)\left(I \otimes D \mathbf{F}\left(z_{t}^{s}\right)-I \otimes \mathbf{M}_{E G} D \mathbf{F}\left(z_{t}^{s}\right)+C \otimes W \mathbf{M}_{E G} D \mathbf{F}\left(X_{t}^{s}\right)\right) \Delta_{t} \\
& =Q \otimes D \mathbf{F}\left(X_{t}^{s}\right)-Q \otimes \mathbf{M}_{E G} D \mathbf{F}\left(X_{t}^{s}\right)+Q C \otimes W \mathbf{M}_{E G} D \mathbf{F}\left(X_{t}^{s}\right) \Delta_{t} \\
& =I \otimes D \mathbf{F}\left(X_{t}^{s}\right)-I \otimes \mathbf{M}_{E G} D \mathbf{F}\left(X_{t}^{s}\right)+\Lambda^{*} \otimes W \mathbf{M}_{E G} D \mathbf{F}\left(X_{t}^{s}\right) Y_{t},
\end{aligned}
$$

pois $\Delta_{t}=(Q \otimes I)^{-1} Y_{t}=\left(Q^{-1} \otimes I\right) Y_{t}$. Desde que $\Lambda^{*}=\operatorname{diag}\left(\lambda_{0}, \lambda_{1}, \ldots, \lambda_{n-1}\right)$, obtemos

$$
\left.Y_{t+1}=\bigoplus_{j=0}^{n-1}\left(I-\mathbf{M}_{E G}+\lambda_{j} W \mathbf{M}_{E G}\right) D \mathbf{F}\left(X_{t}^{s}\right)\right) Y_{t},
$$

onde $\bigoplus$ representa a soma direta.

A importância da decomposição por blocos em (9) está no fato que a estabilidade local do estado sincronizado do sistema (4) pode ser estudado analisando o espectro dos blocos individuais de (9). A matriz $C$ é duplamente estocástica (todas as linhas e todas as colunas somam 1), por aplicação do Teorema de Gershgorin, temos que $\lambda_{0}=1$ é o autovalor dominante de $C$. O bloco correspondendo a tal autovalor corresponde a matriz variacional do sistema de equações (5) que gera a trajetória sincronizada, enquanto os outros $n-1$ blocos correspondem às direções transversais do estado sincronizado e governam sua estabilidade assintótica local. De modo a descrever o comportamento do atrator sincronizado e sua estabilidade assintótica local, definimos o número paralelo de Lyapunov, $h$, por

$$
h\left(z_{0}^{s}\right)=\lim _{\tau \rightarrow \infty}\left\|P_{0, \tau-1} \cdot \ldots \cdot P_{0,1} P_{0,0}\right\|^{1 / \tau},
$$

onde $P_{0, t}=\left(I-\mathbf{M}_{E G}+W \mathbf{M}_{E G}\right) D \mathbf{F}\left(X_{t}^{s}\right)$, e o maior número transversal de Lyapunov, $K$, por

$$
K\left(X_{0}^{s}\right)=\max _{j=1, \ldots, n-1}\left(\lim _{\tau \rightarrow \infty}\left\|P_{j, \tau-1} \cdot \ldots \cdot P_{j, 1} P_{j, 0}\right\|^{1 / \tau}\right),
$$

onde $\left.P_{j, t}=\left(I-\mathbf{M}_{E G}+\lambda_{j} W \mathbf{M}_{E G}\right)\right) D \mathbf{f}\left(X_{t}^{s}\right), j=1,2, \ldots, n-1$, para todos pontos iniciais $X_{0}^{s}$.

Seja $\rho$ medida natural de probabilidade para $\mathbf{g}$, onde $\mathbf{g}: R^{d p} \rightarrow R^{d p}$ é dada por $\mathbf{g}(z)=$ $\left(I-\mathbf{M}_{E G}+W \mathbf{M}_{E G}\right) \mathbf{F}(X)$, ou seja, o mapeamento que gera o atrator sincronizado dado na equação (5). Supondo a integrabilidade de $l n^{+}\left\|\left[I-\mathbf{M}_{E G}+\lambda_{j} W \mathbf{M}_{E G}\right] D \mathbf{F}(X)\right\|$ com a ergodicidade de $\rho$, podemos aplicar o teorema Ergódico de Oseledec [3] para garantir a existência e unicidade dos limites definindo $h$ em (10) e $K$ em (11) e estabelecer um critério para a estabilidade assintótica de um atrator contido na variedade invariante sincronizada por

$$
K<1 \text {. }
$$

O valor $h$ está associado ao atrator sincronizado e trajetórias caóticas são observadas se $h>1$, enquanto trajetórias periódicas para $h<1$. 


\section{Aplicações e Simulações Numéricas}

Quando espécies interagem, a dinâmica de cada espécie é afetada. O uso de modelos para entender as interações entre espécies, tais como presa-predador ou hospedeiro-parasitóide, foi inicialmente feito por Vito Volterra [10]. A motivação foi que as oscilações observadas em populações de peixes poderiam ser explicadas formulando equações diferenciais que explicassem essa interação entre presas e predadores.

Um modelo de equações discretas que analisam a interação de hospedeiros e parasitóides foi desenvolvido por Nicholson e Bailey [7]. Nesse modelo, temos um ponto fixo positivo que é instável. Um número de fatores estabilizantes foram impostos sobre o modelo de modo a estabilizar a coexistência de espécies nesses sistemas [1,4]. Um modelo que apresenta coexistência de hospedeiros e parasitóides é dado por

$$
\begin{aligned}
& N_{t+1}=N_{t} \exp \left(r\left(1-\frac{N_{t}}{k}\right)-a P_{t}\right) \\
& P_{t+1}=c N_{t}\left(1-\exp \left(-a P_{t}\right)\right),
\end{aligned}
$$

onde $N_{t}$ é o número de hospedeiros e $P_{t}$ é o número de parasitóides no passo de tempo $t$. A constante $c$ representa o número médio de novos parasitóides gerados por hospedeiro e $a$ é uma constante que determina a eficiência dos parasitóides. Podemos verificar que o sistema de equações acima possui 3 equilíbrios: $(0,0),(k, 0)$ e $\left(N^{*}, P^{*}\right)$, onde $P^{*}=\frac{r}{a}\left(1-\frac{N^{*}}{k}\right)$ e $N^{*}$ satisfaz $\frac{r}{a c N^{*}}\left(1-\frac{N^{*}}{k}\right)=1-\exp \left(-r\left(1-\frac{N^{*}}{k}\right)\right)$. Os pontos de equilíbrio $(0,0)$ e $(k, 0)$ são instáveis, enquanto a estabilidade de $\left(N^{*}, P^{*}\right)$ depende dos parâmetros do modelo [1]. Além disso, o sistema de equações (13) pode apresentar variados comportamentos, ver figura 2. Nessa figura, apresentase o valor do número de Lyapunov, $L$, de (13). Trajetórias periódicas são caracterizadas para $L<1$, enquanto as caóticas para $L>1$.
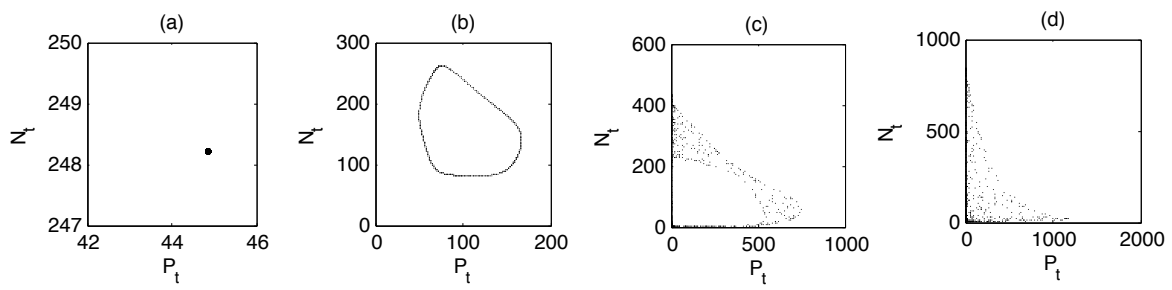

Figura 2: Comportamento do sistema hospedeiro parasitóide com $a=0,01$. (a) $r=2, c=0,5$, $L=0,85$. (b) $r=2, c=1,1, L \approx 1$. (c) $r=2, c=3, L=1,105$. (d) $r=3,1, c=3, L=1,368$.

Apresentamos um caso particular do modelo onde indivíduos migram preferencialmente para um determinado sítio no grupo vizinho. A dinâmica local de cada sítio é dada por (13) e há dois sítios em cada grupo, sem migração entre eles. Além disso, consideramos os grupos de sítios conectados com os dois vizinhos mais próximos em forma de anel com uma dispersão simétrica, ou seja, $50 \%$ de indivíduos para cada grupo. Assim, a matriz $C$ é inversível e seus autovalores são dados por $\lambda_{0}=1$ e $\lambda_{j}=\cos \left(\frac{2 \pi j}{n}\right), j=1,2, \ldots, n$.

Durante a migração entre os grupos, supomos que tanto os hospedeiros quanto os parasitóides migram preferencialmente para o sítio de número 1 no grupo de chegada. Nesse caso, a matriz de distribuição é dada por

$$
W=\left(\begin{array}{llll}
1 & 0 & 1 & 0 \\
0 & 1 & 0 & 1 \\
0 & 0 & 0 & 0 \\
0 & 0 & 0 & 0
\end{array}\right) .
$$

Portanto, o sistema de equações (5) pode ser escrito como

$$
\begin{array}{ccc}
N_{t+1}^{1, s}= & N_{t}^{1, s} \exp \left(r\left(1-\frac{N_{t}^{1, s}}{k}\right)-a P_{t}^{1, s}\right)+\mu_{N} N_{t}^{2, s} \exp \left(r\left(1-\frac{N_{t}^{2, s}}{k}\right)-a P_{t}^{2, s}\right) \\
P_{t+1}^{1, s}= & c N_{t}^{1, s}\left(1-\exp \left(-a P_{t}^{1, s}\right)\right)+\mu_{p} c N_{t}^{2, s}\left(1-\exp \left(-a P_{t}^{2, s}\right)\right) \\
N_{t, 1}^{2, s}= & \left(1-\mu_{N}\right) N_{t}^{2, s} \exp \left(r\left(1-\frac{N_{t}^{2, s}}{k}\right)-a P_{t}^{2, s}\right) \\
P_{t+1}^{2, s}= & \left(1-\mu_{p}\right) c N_{t}^{2, s}\left(1-\exp \left(-a P_{t}^{2, s}\right)\right) .
\end{array}
$$


onde $N_{t}^{i, s}$ e $P_{t}^{i, s}$, representam os hospedeiros e parasitóides em cada sítio $i, i=1,2$.

A Figura 3 mostra o comportamento das trajetórias sincronizadas em função do parâmetro $\mu$, onde $\mu:=\mu_{N}=\mu_{P}$. Em (a), observa-se extinção no sítio 2 e isso é caracterizado por altas taxas de migração. Em (b), (c) e (d), observa-se uma região onde as trajetórias convergem para um ponto fixo caracterizando um efeito estabilizador. Além disso, devido ao movimento preferencial, os atratores sincronizados tendem a ter mais densidade populacional no sítio 1 para qualquer valor da fração de migração. Observe que esse efeito estabilizante é caracterizado por ciclos limites que diminuem sua circunferência à medida que a taxa de migração é aumentada até convergir a um ponto fixo (Figura 4 coluna (iii), (c) e (d)). A Figura 4 mostra o maior número tranversal de Lyapunov. Observamos que trajetórias sincronizadas periódicas são estáveis (Figura 4 (a) e (b)). Enquanto os casos onde a dinâmica local de cada grupo exibe caos, as trajetórias sincronizadas dependem da fração de dispersão (Figura 4 (c) e (d)).

(a)

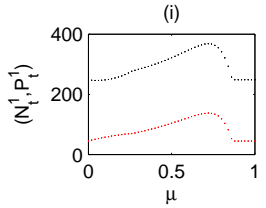

(b)

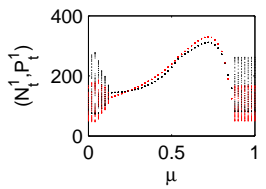

(c)

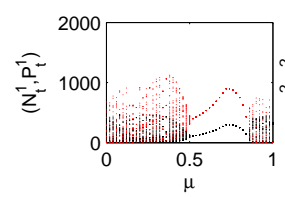

(d)

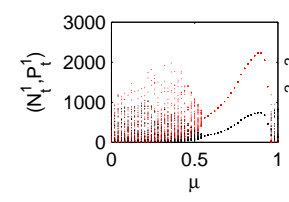

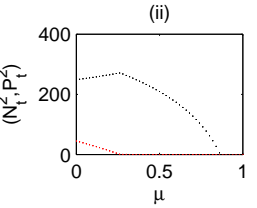
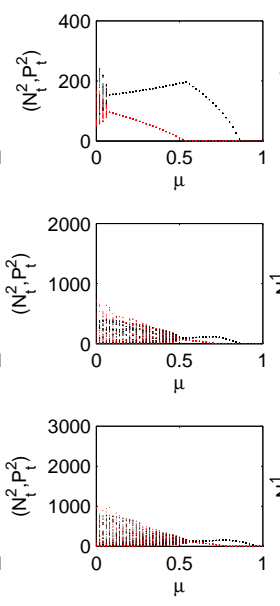
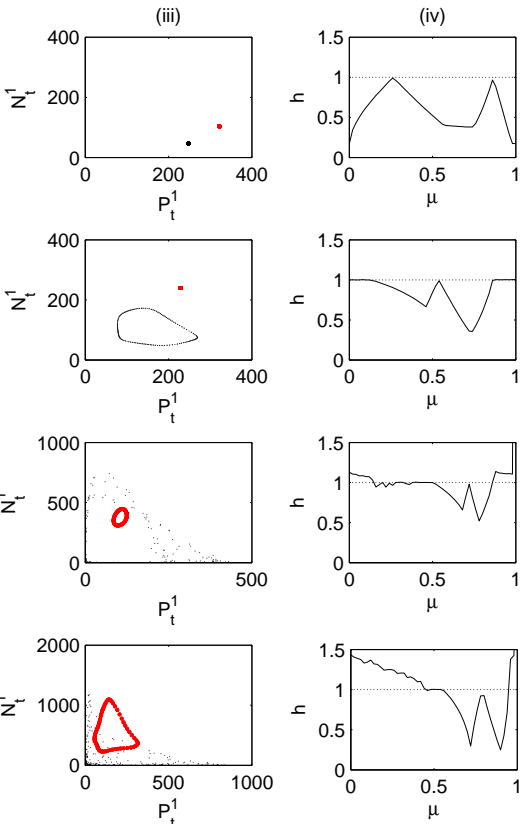

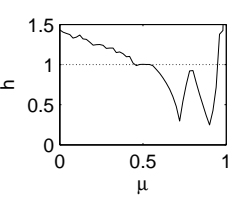

Figura 3: Comportamento do atrator sincronizado para dois sítios com distribuição preferencial e $a=0,01$. (a) $r=2, c=0,5$. (b) $r=2, c=1,1$. (c) $r=2, c=3$. (d) $r=3,1, c=3$. (i) $N_{t}^{1}$ em preto e $P_{t}^{1}$ em vermelho. (ii) $N_{t}^{2}$ em preto e $P_{t}^{2}$ em vermelho. (iii) $N_{t}^{1}$ vs $P_{t}^{1}$ para $\mu=0,01$ em preto e $\mu=0,5$ em vermelho. (iv) Número paralelo de Lyapunov vs $\mu$.
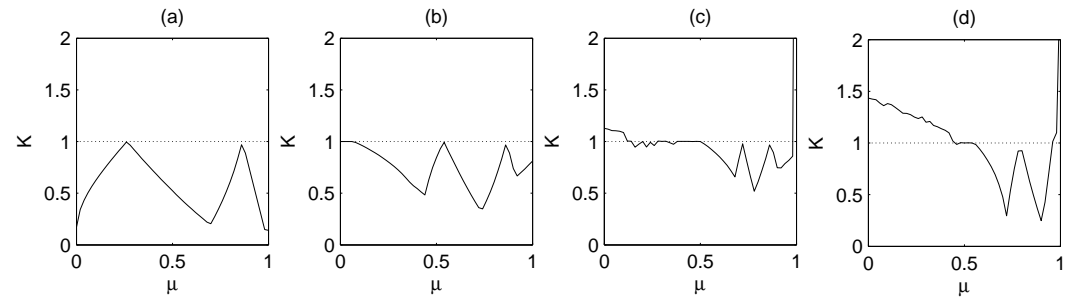

Figura 4: Número transversal de Lyapunov considerando 2 sítios em cada grupo e distribuição preferencial vs $\mu$. Considera-se 5 grupos acoplados com os dois vizinhos mais próximos em forma de anel e $a=0,01$. (a) $r=2, c=0,5$. (b) $r=2, c=1,1$. (c) $r=2, c=3$. (d) $r=3,1, c=3$. 


\section{Discussão}

Apresentamos uma rede de sítios com multi-espécies e sitribuídos em duas escalas geográficas. $\mathrm{Na}$ escala local, os indivíduos migram entre os sítios que compõem um grupo por processo de curta dispersão. Na escala regional, os indivíduos migram entre os grupos por processo de longa dispersão. Analisamos o fenômeno de sincronização entre os grupos e obtemos um critério analítico para a estabilidade assintótica de trajetórias sincronizadas baseado no cálculo dos números de Lyapunov. O cálculo do critério é obtido através da decomposição em blocos da matriz Jacobiana que nos permite descrever o comportamento das trajetórias sincronizadas e de sua estabilidade assintótica. O ponto chave é que um dos blocos corresponde a matriz variacional que gera as trajetórias sincronizadas. Desse bloco, calculamos o número paralelo de Lyapunov, $h$, que informa o comportamento das trajetórias sincronizadas, ou seja, periódica $(h<1)$ ou caótica $(h>1)$. Os demais blocos correspondem às direções transversais e deles calculamos o maior número transversal de Lyapunov, $K$, que informa quando órbitas que inciam próximas ao estado sincronizado são atraídas $(K<1)$ ou repelidas $(K>1)$ a ele.

As simulações numéricas mostram as diferentes trajetórias sincronizadas geradas pelo processo migratório. Na situação onde o modelo local de cada sítio considera a interação entre hospedeiros e parasitóides e indivíduos migram para os grupos vizinhos e se distribuem preferencialmente num determinado sítio, o comportamento das trajetórias sincronizadas apresentam um efeito estabilizante a medida que a taxa de migração é aumentada (Figura 3) e a estabilidade de tais trajetórias depende do processo migratório (Figura 4). Obviamente, outras dinâmicas locais e outras formas de distribiução poderiam ser consideradas, mas os resultados apresentadas informam fatores relevantes devido ao processo migratório e de como os indivíduos se distribuem nos diferentes grupos de sítios.

\section{Referências}

[1] J. R. Beddington, C. A. Free, J. H. Lawton, Dynamic complexity in predator-prey models framed in difference equations, Nature, 225 (1975) 58-60.

[2] D. J. Earn, S. A. Levin, P. Rohani, Coherence and Conservation, Science, 290 (2000) 13601364 .

[3] J. P. Eckmann, D. Ruelle, Ergodic Theory of chaos and strange attractors, Rev. Modern Phys., 57 (1985) 617-656.

[4] I. Hanski, Spatial patterns of coexistence of competing species in patchy habitats. Theoretical Ecology, 1 (2008) 29-43.

[5] M. P. Hassell, H. N. Comins, R. M. May, Species coexistence and self-organizing spatial dynamics Nature, 370 (1994) 290-292.

[6] V. A. A. Jansen, A. L. Lloyd, Local stability analysis of spatially homogeneous solutions of multi-patch systems, J. Math. Biol. 41 (2000), 232-252.

[7] A. J. Nicholson, V. A. Bailey, The balance of animal populations. Proc Zool Lond, (1935) $551-598$.

[8] P. Rohani, R. M. May, M. P. Hassel, Metapopulations and Equilibrium Stability: The Effects of Spatial Structure, J. theor. Biol., 181 (1996) 97-109.

[9] P. Rohani, G. D. Ruxton, Dispersal-Induced Instabilities in Host-Parasitoid Metapopulations, Theoretical Population Biology, 55 (1999) 23-36.

[10] V. Volterra, Fluctuations in the abundance of a species considered mathematically, Nature, 118 (1926) 558-560. 